\title{
Alzheimer's imaging agents struggle to find a market outside trials
}

Physicians in the US who want to see how much amyloid-beta protein has accumulated in the brains of their patients with Alzheimer's disease now have two options of imaging agents. On 25 October, the US Food and Drug Administration (FDA) announced the approval of Vizamyl (flutemetamol F-18), a positron emission tomography (PET) radiotracer that binds to amyloid plaques, the abnormal brain deposits that are thought to cause neurodegeneration. Vizamyl, developed by UK-headquartered GE Healthcare, joins a similar diagnostic compound for Alzheimer's disease called Amyvid (florbetapir F-18), which received approval last year.

"I think there should be a great deal of enthusiasm with the fact that we're gotten these agents to the point where they're FDAapproved," says David Wolk, a neurologist at the University of Pennsylvania in Philadelphia who was involved in clinical trials of Vizamyl. "Having more options will certainly move the field forward."

However, these new options still have their limitations. Both imaging agents have been granted only narrow approval: the compounds can't be used to definitively diagnose Alzheimer's disease or to gauge treatment effectiveness, only to support other diagnostic criteria.

Some studies have found amyloid buildup in elderly people with all their mental faculties; others have shown that the toxic protein accumulates in other forms of dementia. So, for now, the FDA has ruled that a negative scan in someone with cognitive impairment can be used to point clinicians toward other causes of dementia, while a positive scan remains more ambiguous.

In light of the limited diagnostic utility of available imaging agents for Alzheimer's, in late September the US Centers for Medicare and Medicaid Services (CMS) issued a final decision not to provide coverage for Amyvid, which is marketed by Philadelphia-based Avid Pharmaceuticals, a subsidiary of Indiana's Eli Lilly. Since most private insurers in the US follow the CMS's lead, the ruling has effectively shut down the commercial market for the class of imaging agents for the time being. (In Europe, Amyvid received a similarly limited marketing authorization from European regulators in early 2013; decisions on coverage in the various EU member states haven't been finalized.)

Without the added market boost of insurance coverage, most people in the field expect Vizamyl to make a difference mainly in clinical trials. But many believe the two new imaging

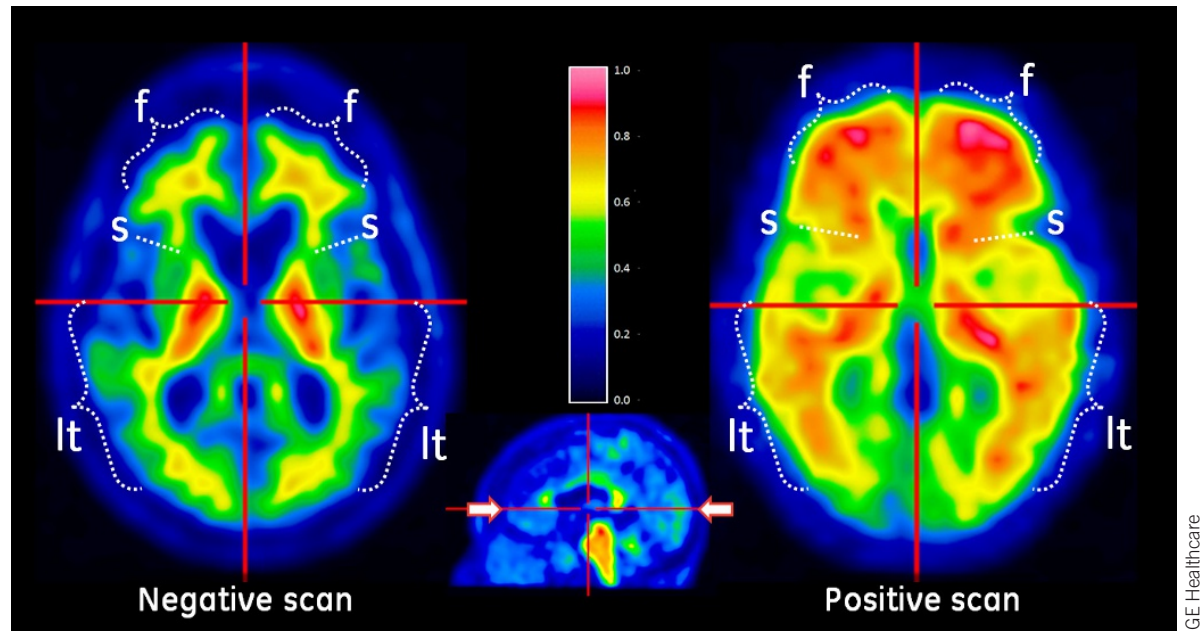

Head to head: Vizamyl PET images of a healthy brain (left) and an Alzheimer's brain (right).

agents are already poised to have a larger impact. In a recently published set of best-practice guidelines on the use of amyloid PET imaging, Dean Hartley, director of science initiatives at the Alzheimer's Association in Chicago, and a team of other scientists from around the US suggest that the imaging agents are wellstudied enough that they can be used in clinical settings to help diagnose patients with early onset of Alzheimer's or unusual symptoms of disease (Alzheimers Dement. 9, e106-e109, 2013). In these cases, the battery of cognitive tests clinicians rely on for diagnose can lead to mixed results, and the scans could help clarify diagnosis, treatment options and prognosis. "We think there is a specific population that can gain from having this ability to detect amyloid," Hartley says.

\section{Scanning the horizon}

Pharmaceutical companies are already moving beyond that suggested use, relying on the imaging agents both to screen patients for inclusion in trials and to track amyloid levels throughout a treatment regimen. For instance, New Jerseybased Merck has included a positive Vizamyl scan in the inclusion criteria for a 1,500-person phase 3 trial involving a drug that blocks the beta-secretase BACE, an enzyme that initiates the production of amyloid-beta. A subanalysis of that trial will also follow the change in such scans throughout treatment. Meanwhile, Eli Lilly is using Amyvid for similar purposes in a pivotal trial testing the amyloid-targeting antibody solanezumab or placebo in 2,100 people with early-stage Alzheimer's disease.

Studies involving imaging agents for Alzheimer's, as they grow in size and length, could ultimately be used to support the approval of the amyloid imaging agents for broader use as diagnostic tools or a way to track disease progression. What's needed to take that step are additional studies that definitively link changes in amyloid to treatment success_-but that will first require successful treatments to be developed. Nevertheless, more companies are hoping to bring imaging agents to market today for the eventuality of wider approval. For example, Ohio-based Navidea Biopharmaceuticals has an imaging agent called NAV4694 in ongoing phase 3 trials, and florbetaben, developed by Berlinbased Piramal Imaging, is currently under review with both US and EU regulators.

All these compounds-variants of a dye called thioflavin T, which binds beta-sheet-rich structures such as amyloid plaques-use the radioisotope fluorine-18 to visualize plaques in a PET scan. As Wolk points out, both of the two approved products, Amyvid and Vizamyl, "are good agents" and both "have fairly high sensitivity for detecting amyloid plaques." The sole noteworthy difference between the two compounds is that Amyvid is FDA-approved for use in black and white scans only, whereas Vizamyl can be developed into images with added false coloring.

According to Ger Brophy, vice president of new product development in the medical diagnostics branch of GE Healthcare in New York, the color scans have no additional data, but they make it easier for radiologists to interpret the images. "We've had hugely positive feedback from the community in terms of the extra information people think they can process," Brophy says. Now, they just have to convince insurers and the medical community that that information is clinically meaningful.

Sarah CP Williams 